Research Article

\title{
Relative Performance Concern on DC Pension Plan under Heston Model with Inflation Risk
}

\author{
Aimin Song $\mathbb{B D}^{1}$ and Peimin Chen $\mathbb{D i D}^{2}$ \\ ${ }^{1}$ School of Economic Mathematics, Southwestern University of Finance and Economics, Chengdu 611130, China \\ ${ }^{2}$ Shanghai Business School, Shanghai 200235, China \\ Correspondence should be addressed to Peimin Chen; pmchen@sbs.edu.cn
}

Received 2 April 2020; Revised 13 September 2020; Accepted 28 November 2020; Published 21 December 2020

Academic Editor: Michele Guida

Copyright (c) 2020 Aimin Song and Peimin Chen. This is an open access article distributed under the Creative Commons Attribution License, which permits unrestricted use, distribution, and reproduction in any medium, provided the original work is properly cited.

\begin{abstract}
With the global outbreak of new coronavirus pneumonia, more and more countries have entered the state of sealing off cities. After the epidemic, with the shortage of some materials, the economy is very likely to enter the state of inflation. Thereby, it is necessary and urgent for us to reconsider investment problems involving inflation risk. In this paper, we mainly study the optimal investment strategy of two defined contribution (DC) pension managers with strategy interaction under inflation risk. The traditional portfolio literatures mainly focus on DC pension plan and try to maximize the expected utility of terminal nominal wealth. In this paper, we consider the more complicated situation that pension managers have, both concerns on relative wealth and relative risk aversion. Then, the objective function is constructed to satisfy these two concerns. The dynamic programming principle method is employed to solve the above problems, and a series of analytical solutions to this problem are obtained. Finally, some numerical examples are discussed for the economic implications to support our theoretical results.
\end{abstract}

\section{Introduction}

As a continuous increase in the average life expectancy of human beings, the optimal portfolio strategies of pension funds have been continuously studied in the literature. Generally speaking, there are two types of pension plans. One is the defined benefit (DB) pension plan, in which people do not need to consider the pension's investment portfolio and longevity risks and enjoy a fixed benefit after retirement. The other one is the defined contribution (DC) pension plan, in which workers pay their pensions at a certain rate of their salary before retirement. Compared with the DB pension plan, DC pension plan relieves the pressure on the social security system by shifting investment risk and longevity risk from sponsors to pension plan members. As a result, more and more countries have transferred partially or even completely from the DB pension plan to the DC pension plan. The purpose of this paper is to discuss the asset allocation of the DC pension plan.
In past decades, many literature studies have studied the optimal investment strategy of DC pension funds. Some researchers start with different utility functions and consider the issue of maximizing the expected utility of the terminal wealth. For instances, Gao [1] studies the optimal investment strategy of DC pension under CRRA and CARA utility function. Guan and Liang [2] discuss the maximization of expected utility of the terminal wealth under loss aversion utility function, which is an S-shaped curve. Vigna [3] uses the embedding technique to solve the mean variance portfolio selection problem in the accumulation phase of a DC pension plan. Moreover, lots of research studies have been carried out based on the characteristics of the pension plan itself. For example, Boulier et al. [4] focus on DC pension plan for a guarantee expressed by the benefits, and the guarantee depends on the level of the stochastic interest rate. Zeng et al. [5] provide an optimal investment strategy for an ambiguity aversion investor, who faces uncertain economic conditions over a long time horizon. Han and Hung [6] use the stochastic dynamic programming principle 
(DPP) to investigate the optimal asset allocation for a DC pension plan with downside protection under stochastic inflation. Guan and Liang [7] find the optimal investment strategy in a stochastic interest rate and stochastic volatility framework.

The works above mainly focus on maximizing the expected utility of terminal wealth. However, in real markets, pension managers not only focus on such an objective but also are very concerned about the relative performance of other pension managers. In fact, economic and sociological studies have emphasized the importance of relative concerns in human behaviors, and they have a very vivid title for this phenomenon with "catching up with the Joneses" (see Veblen [8]; Abel [9]; Gali [10]; Gomez [11]). Later, some literature discusses the relative wealth concern of asset portfolios. In [12], it is the first time to discuss the optimal investment strategy under the wealth correlation between two investors in the framework of stochastic dynamic investment games in continuous time. Espinosa and Touzi [13] consider a continuous time stochastic dynamic model and the optimal investment strategy of two investors with relative wealth concern. For terminal wealth, the author does not just consider the investors' own wealth, but cover the convex combination with respect to the wealth of the investors and their competitors. The optimal portfolio problem is solved by using the DPP method. Basak and Makarov [14] consider a dynamic model in incomplete market for two managers with CARA utility function. They obtain a unique Nash equilibrium strategy and provide the equilibrium portfolio policies explicitly. Guan and Liang [15] study a portfolio game with stochastic Nash equilibrium between two risk aversion managers under inflation risk.

The literature above only assume that the investors are only concerned about the relative wealth about their competitors, but in real investment, markets investors not only care about this point but also are affected by the risk aversion of competitors. This consideration is especially necessary when we consider the competitors as the representative manager in the market. Therefore, in this paper, we incorporate the relative risk aversion concern into the model. That is, the relative performance concern (RPC) covers both relative wealth concern (RWC) and relative risk aversion concern (RRAC). In this paper, the previous works would be a special case of our model. As far as we known, there are no case of incorporating risk aversion into the utility function yet.

We verify that the model established in this paper can be analyzed as a model between two ordinary managers of pension funds or an ordinary pension fund manager and a representative manager in the pension funds market. Since the investment on pension funds generally takes a long time, inflation risk as an important long-term risk cannot avoid such an investment. So, we incorporate inflation risk in our model. In this paper managers can invest on an inflationlinked index bond (IIB) to reduce the inflation risk of pension funds. We also assume that stocks follow the Heston process. By the DPP method and the corresponding HJB equation, we obtain the explicit solution of the optimal portfolio strategies of RPC manager and the specific solution for the cases of RWC and RRAC, respectively. Comparing with Merton manager, we find that RWC manager always tends to invest more shares on stocks. Moreover, we compare the optimal strategy of RPC, RWC manager with Merton manager and find that both RPC and RWC are essentially a distortion of the managers' risk aversion coefficient with Merton optimization strategy. These findings, to the best of our knowledge, have not been reported or identified in the previous literature.

The rest of this paper is arranged as follows. Section 2 introduces the financial markets, which include a risk free asset, stock, and IIB. Section 3 makes the main result of our article; we use the DPP and stochastic optimal control method to solve the optimal portfolio and discus several special cases. In Section 4, we gave a numerical analysis based on the main conclusions of this paper, combined with market data, and we have analyzed its economic significance. In Section 5, we summarized the main points and conclusions of the article.

\section{Mathematical Models}

Let $\left(\Omega, \mathscr{F}, \mathscr{F}_{t}, P\right)$ be a complete filtering probability space. Assume that all random variables and stochastic processes are well defined and adapted to $\left\{\mathscr{F}_{t}, t \in[0, T]\right\}$ in this article.

2.1. Financial Models. Broadly speaking, DC pension funds have a long investment cycle. Inflation risk has a great impact on the real value of DC pension funds. In this paper, we incorporate the inflation risk into our model. In economy, the consumer price index (CPI) is often regarded as an important indicator of inflation. Following Brennan and Xia [16] and Kwak and Lim [17], we assume that the consumer price process satisfies the following diffusion process:

$$
\left\{\begin{array}{l}
\frac{\mathrm{d} P(t)}{P(t)}=\mu_{p} \mathrm{~d} t+\sigma_{p} \mathrm{~d} W_{p}(t), \\
P(0)=P_{0},
\end{array}\right.
$$

where $P(t)$ is the price index, and it refers to the purchase power per unit of money, $\mu_{p}$ and $\sigma_{p}>0$ are the instantaneous expectation and volatility of inflation rate, and $W_{p}(t)$ is a standard Brownian motion, which represents the risk sources of inflation.

In this paper, in financial market, we invest three types of assets, cash, inflation-linked index bond (IIB), and stock. The price of the risk-free (i.e., cash) asset $S_{0}(t)$ follows the process as

$$
\frac{\mathrm{d} B(t)}{B(t)}=r_{n} \mathrm{~d} t
$$

where $r_{n}$ denotes the nominal interest rate.

As described in Sun et al. [18], the process of IIB is supposed to follow

$$
\frac{\mathrm{d} I(t)}{I(t)}=r \mathrm{~d} t+\frac{\mathrm{d} P(t)}{P(t)}=\left(r+\mu_{p}\right) \mathrm{d} t+\sigma_{p} \mathrm{~d} W_{p}(t),
$$

where $r$ is the real interest rate and $r+\mu_{p}$ represents the expected yield of inflation bonds. 
In addition, because the inflation rate usually has a direct or indirect impact on the price of stocks (see Lee [19]), in this paper we assume that the price of stock is affected not only by its own risk source $W_{s}(t)$ but also by inflation risk $W_{p}(t)$. The stock prices $S(t)$ are supposed to follow the Heston model with the inflation risk as follows:

$$
\left\{\begin{array}{l}
\frac{\mathrm{d} S(t)}{S(t)}=r_{n} \mathrm{~d} t+\sqrt{V(t)}\left(\lambda_{s} \sqrt{V(t)} \mathrm{d} t+\mathrm{d} W_{s}(t)\right)+\sigma_{s}\left(\lambda_{p} \mathrm{~d} t+\mathrm{d} W_{p}(t)\right)=\left[r_{n}+\lambda_{s} V(t)+\sigma_{s} \lambda_{p}\right] \mathrm{d} t+\sqrt{V(t)} \mathrm{d} W_{s}(t)+\sigma_{s} \mathrm{~d} W_{p}(t) \\
\mathrm{d} V(t)=\kappa[\delta-V(t)] \mathrm{d} t+\sigma_{v} \sqrt{V(t)} \mathrm{d} W_{v}(t) \\
V(0)=v
\end{array}\right.
$$

where $\kappa, \delta, \sigma_{v}, \lambda_{s}$, and $\sigma_{s}$ are all positive constants, $\sigma_{s}$ and $\sqrt{V(t)}$ represent the volatilities generated by the movements of inflations and stock prices, and $\lambda_{p}$ and $\lambda_{s} \sqrt{V(t)}$ represent the corresponding market prices of risk, and $W_{s}(t)$ and $W_{v}(t)$ are two standard Brownian motions. We further assume that $W_{s}(t)$ and $W_{v}(t)$ are independent with $W_{p}(t)$, respectively, while $W_{s}(t)$ and $W_{v}(t)$ are dependent with $E\left[W_{s}(t) W_{v}(t)\right]=\rho t$. Here, $\rho \in[-1,1]$. Moreover, we need a condition $2 \kappa \delta>\sigma_{v}^{2}$ to ensure $V(t)>0$.

Remark 1. Define $\mathbb{Q}$ as a risk neutral measure. From no arbitrage theory, then the drift term of stochastic differential equation 3 can be rearranged as follows:

$$
r+\mu_{p}-\sigma_{p} \lambda_{p}=r_{n}
$$

In Section 3.1, we will use this conclusion to get the compact form of real wealth process.

2.2. The Pension Management. Suppose that, in the pension market, there are two pension managers. They can invest three assets: IIB, stock, and cash. Assume that the proportion of investment on these three assets is $\pi_{I i}(t), \pi_{S i}(t)$, and $1-\pi_{I i}(t)-\pi_{S i}(t)(i=1,2)$, respectively. On the contrary, pension funds also receive regular contributions. To be more realistic, we assume that contributions increase as the price index grows. That is, contributions follow $c_{i} P(t)$, where $c_{i}>0(i=1,2)$ is a parameter. Suppose that there is no transaction cost and taxes in financial market and short sell is allowed. Under these assumptions, the wealth process can be expressed as

$$
\mathrm{d} X_{i}(t)=c_{i} P(t) \mathrm{d} t+X_{i}(t)\left[\left(1-\pi_{I i}(t)-\pi_{S i}(t)\right) \frac{\mathrm{d} B(t)}{B(t)}+\pi_{I i}(t) \frac{\mathrm{d} I(t)}{I(t)}+\pi_{S i}(t) \frac{\mathrm{d} S(t)}{S(t)}\right]
$$

where $X_{i}(0)=x_{i}, \quad x_{i} \geq 0(i=1,2)$ represents the initial wealth of pension manager $i$. Substituting (2)-(4) into equation (6), we can obtain the following wealth process for pension manager $i$ :

$$
\left\{\begin{array}{l}
\mathrm{d} X_{i}(t)=c_{i} P(t) \mathrm{d} t+r_{n} X_{i}(t) \mathrm{d} t+\pi_{I i}(t) X_{i}(t)\left[\left(r+\mu_{p}-r_{n}\right) \mathrm{d} t+\sigma_{p} \mathrm{~d} W_{p}(t)\right] \\
+\pi_{S i}(t) X_{i}(t) \sqrt{V(t)}\left[\lambda_{s} \sqrt{V(t)} \mathrm{d} t+\mathrm{d} W_{s}(t)\right]+\pi_{S i}(t) X_{i}(t) \sigma_{s}\left[\lambda_{p} \mathrm{~d} t+\mathrm{d} W_{p}(t)\right] \\
X_{i}(0)=x_{i} .
\end{array}\right.
$$

Let $\pi_{i}(t)=\left(\pi_{I i}, \pi_{S i}\right)^{\top}$ denote the investment strategy of pension manager $i$. In the following, firstly, we present the definition of admissible strategy.

Definition 1. Let $\mathcal{O}:=\mathbb{R} \times \mathbb{R}^{+}$and $\mathscr{G}:=[0, T] \times \mathcal{O}$. An investment strategy is called an admissible strategy if it meets the following conditions:

(1) $\pi_{i}(t)$ is $\mathscr{F}_{t}$-predictable

(2) For any $(x, v) \in \mathcal{O}$, the stochastic differential equation (7) has a unique solution with $X_{i}(t)=x_{i}, V(t)=v$
(3) $E\left\{\int_{0}^{T}\left[\pi_{\mathrm{Ii}}^{2}(t) \sigma_{p}^{2}+\pi_{\mathrm{Si}}^{2}(t)+\pi_{\mathrm{Si}}^{2}(t) \sigma_{s}^{2}\right] \mathrm{d} t\right\}<\infty$

We denote the set of all admissible strategy by $\mathscr{A}_{i}$. In this paper, we are trying to find the optimal investment strategy for two DC pension plan managers under admissible strategy $\mathscr{A}_{i}$.

\section{The Competition Model}

3.1. Nonzero-Sum Stochastic Differential Game. In this section, we consider a random differential game model with nonzero sum for two managers. The differential game 
problem is constructed based on two relative performance processes and the utility maximization problem at the fixed terminal time. We use $Y_{i}(T)=\left(X_{i}(T) / P(T)\right)(i=1,2)$ to represent the real wealth process of manager $i$, which can be expressed as follows according to (7):

$$
\begin{aligned}
\mathrm{d} Y_{i}(t)= & c_{i} \mathrm{~d} t+r Y_{i}(t) \mathrm{d} t+\left(\pi_{I i}-1\right)(t) Y_{i}(t) \sigma_{p}\left[\left(\lambda_{p}-\sigma_{p}\right) \mathrm{d} t+\mathrm{d} W_{p}(t)\right] \\
& +\pi_{S i}(t) Y_{i}(t) \sigma_{s}\left[\left(\lambda_{p}-\sigma_{p}\right) \mathrm{d} t+\mathrm{d} W_{p}(t)\right]+\pi_{S i}(t) Y_{i}(t) \sqrt{V}\left[\lambda_{s} \sqrt{V} \mathrm{~d} t+\mathrm{d} W_{s}(t)\right] .
\end{aligned}
$$

Let $\tilde{\pi}_{i}(t)=\left(\pi_{I i}(t) \text { and } \pi_{S i}(t)\right)^{\top}-(1,0)^{\top}$. We call $\tilde{\pi}_{i}(t)$ an admissible strategy if $\left(\pi_{I i}(t), \pi_{S i}(t)\right) \in \mathscr{A}_{i}$. For simplicity, we denote $\widetilde{\mathscr{A}}_{i}=\left\{\widetilde{\pi}_{i}(t) \mid\left(\pi_{I i}(t), \pi_{S i}(t)\right) \in \mathscr{A}_{i}\right\}$. Then, by using the result of Remark 1, we can rewrite the evolution of real wealth process $Y_{i}(t)$ as follows:

$$
\mathrm{d} Y_{i}(t)=c_{i} \mathrm{~d} t+r(t) Y_{i}(t) \mathrm{d} t+Y_{i}(t) \tilde{\pi}_{i}(t)^{\top} \Sigma[\Lambda \mathrm{d} t+\mathrm{d} W(t)],
$$

where

$$
\begin{aligned}
\Sigma & =\left[\begin{array}{cc}
\sigma_{p} & 0 \\
\sigma_{s} & \sqrt{V}
\end{array}\right], \\
\Lambda & =\left[\begin{array}{c}
\lambda_{p}-\sigma_{p} \\
\lambda_{s} \sqrt{V}
\end{array}\right], \\
W(t) & =\left[\begin{array}{c}
W_{p}(t) \\
W_{s}(t)
\end{array}\right] .
\end{aligned}
$$

Generally, the managers care about two factors: the amount of money for investment and the relative performance of another manager. Here, the relative performance concerns two aspects. One is that pension manager always care about their competitor's wealth in the financial market because this will lead to a relative ranking in the pension market. The higher the ranking is, usually, the higher the amount of pension contributor will choose his/her pension funds, which will provide the pension manager higher management fee. Another aspect is that managers always concern about the risk aversion horizon of their competitor, as they are inevitably infected by their competitor's risk aversion, especially when the competitor manager is the representative manager of the pension market.

In order to more intuitively study the impact of RPC on the manager's optimal investment strategy, we start with the simplest linear relationship and establish a manager's model of RWC and RRAC. Take manager 1 as an example, we can let $\left(1-K_{1}\right) Y_{1}(t)$ stand for the benefit from his/her real wealth and $K_{1}\left[Y_{1}(t)-Y_{2}(t)\right]$ stand for benefit from relative wealth concern. Similarly, the risk aversion of manager 1 comes from two aspects. We let $\left(1-k_{1}\right) \gamma_{1}$ stand for the risk aversion from manager 1 , where $\gamma_{1}$ means the relative risk aversion of manager 1 before the competition. Let $k_{1} \gamma_{2}$ stand for the weight of risk aversion of manager 2 . Thus, during the competition, the risk aversion of manager 1 is $\left(1-k_{1}\right) \gamma_{1}+k_{1} \gamma_{2}$. Using the same method, we can get the $\mathrm{RWC}$ form and RRAC form of manager 2 . In the following, we just consider manager 1 only, manager 2 can be dealt in the similar way.

Let

$$
\begin{aligned}
Z_{1}(t) & :=\left(1-K_{1}\right) Y_{1}(t)+K_{1}\left(Y_{1}(t)-Y_{2}(t)\right) \\
& =Y_{1}(t)-K_{1} Y_{2}(t) .
\end{aligned}
$$

Then, it follows that

$$
\begin{aligned}
\mathrm{d} Z_{1}(t)= & \mathrm{d} Y_{1}(t)-K_{1} \mathrm{~d} Y_{2}(t) \\
= & \left(c_{1}-K_{1} c_{2}\right) \mathrm{d} t+r(t) Z_{1}(t) \mathrm{d} t \\
& +\left[Y_{1}(t) \tilde{\pi}_{1}(t)-K_{1} Y_{2}(t) \tilde{\pi}_{2}(t)\right]^{\top} \Sigma[\Lambda \mathrm{d} t+\mathrm{d} W(t)] .
\end{aligned}
$$

The parameter $K_{i} \in[0,1]$ is a weight that describes the degree to which managers value the relevant rankings with competitors. Meanwhile, we need to pay attention to two special cases. At $K_{i}=0$, the differential game model degenerates to the optimal investment problem for manager with his/her own terminal real wealth. For $K_{i}=1$, it corresponds to the effect that managers only consider the relative wealth.

Let $U: R^{+} \longrightarrow R$ represent the utility preferences of managers. The optimal goal of the pension manager is to maximize $U(Z(T))$. Assume that the utility functions are strictly concave functions. That is, $U^{\prime}>0$ and $U^{\prime \prime}<0$. Furthermore, the utility function satisfies the following Inada conditions:

$$
\begin{aligned}
& U^{\prime}(-\infty):=\lim _{Z \longrightarrow-\infty} U^{\prime}(Z)=+\infty \\
& U^{\prime}(+\infty):=\lim _{Z \longrightarrow+\infty} U^{\prime}(Z)=0 .
\end{aligned}
$$

We can use constant absolute risk aversion (CARA) function to define the utility function $U(\cdot)$ as

$$
U(z)=-\frac{1}{\gamma} e^{-\gamma z}, \quad \gamma>0 .
$$

3.2. The Nash Equilibrium Strategy. In this section, we use the DPP method to solve this competition problem. If the admissible strategy of manager 2 is given, manager 1 chooses his admissible strategy $\tilde{\pi}_{1} \in \widetilde{\mathscr{A}}_{1}$ to maximize his value function $H_{1}^{\left(\tilde{\pi}_{1}, \tilde{\pi}_{2}\right)}\left(t, z_{1}, v\right)$, and vice versa. The game between two managers will finish at terminal time $T$. The nonzerosum stochastic differential portfolio game can be constructed to maximize the utility of two best investment combinations by 


$$
\begin{aligned}
& H_{1}\left(t, z_{1}, v\right)=\sup _{\tilde{\pi}_{1} \in \widetilde{\mathscr{A}}_{1}} H_{1}^{\left(\tilde{\pi}_{1}, \tilde{\pi}_{2}^{*}\right)}\left(t, z_{1}, v\right), \\
& H_{2}\left(t, z_{2}, v\right)=\sup _{\tilde{\pi}_{2} \in \widetilde{\mathscr{A}}_{2}} H_{2}^{\left(\tilde{\pi}_{1}^{*}, \tilde{\pi}_{2}\right)}\left(t, z_{2}, v\right) .
\end{aligned}
$$

In a dynamic noncooperative problem, the relevant solution concept is relative to Markov perfect Nash equilibrium (MPNE). Here, we present the conception of two player games under MPNE.

Definition 2. The MPNE of two player game is a pair of admissible strategies $\left(\tilde{\pi}_{1}^{*}, \tilde{\pi}_{2}^{*}\right) \in \mathscr{\mathscr { A }}_{1} \times \widetilde{\mathscr{A}}_{2}, z_{1}, z_{2}>0$, such that, for all $\tilde{\pi}_{1} \in \mathscr{\mathscr { A }}_{1}$ and $\widetilde{\pi}_{2} \in \widetilde{\mathscr{A}}_{2}$, the following inequalities hold:

$$
\begin{aligned}
& H_{1}^{\left(\tilde{\pi}_{1}^{*}, \tilde{\pi}_{2}^{*}\right)}\left(t, z_{1}, v\right) \geq H_{1}^{\left(\tilde{\pi}_{1}, \tilde{\pi}_{2}^{*}\right)}\left(t, z_{1}, v\right), \\
& H_{2}^{\left(\tilde{\pi}_{1}^{*}, \tilde{\pi}_{2}^{*}\right)}\left(t, z_{2}, v\right) \geq H_{2}^{\left(\tilde{\pi}_{1}^{*}, \tilde{\pi}_{2}\right)}\left(t, z_{2}, v\right) .
\end{aligned}
$$

In the following, we will derive the form of the MPNE of this pension game. The target function of manager 1 is given by

$$
\begin{aligned}
H_{1}\left(t, z_{1}, v\right) & =\sup _{\tilde{\pi}_{1}(t)} E\left[U_{1}\left(Z_{1}(T)\right) \mid Z_{1}(t)\right. \\
& \left.=z_{1}, V(t)=v\right] .
\end{aligned}
$$

Then, using the DPP method and It $\widehat{o}$ formula, we can derive the HJB equation in the following theorem.
Theorem 1. Assume that $H_{1} \in C^{1,2,2}\left[[0, T] \times \mathbb{R}^{+} \times \mathbb{R}^{+}\right]$ satisfies (17), then the associated $H J B$ equation of $H_{1}$ can be given by

$$
\begin{array}{r}
\sup _{\tilde{\pi}_{1}(t)}\left\{H_{1 t}+H_{1 z_{1}}\left[\left(c_{1}-K_{1} c_{2}\right)+r z_{1}+\left(Y_{1} \tilde{\pi}_{1}-K_{1} Y_{2} \tilde{\pi}_{2}\right)^{\top} \Sigma \Lambda\right]\right. \\
+\frac{1}{2} H_{1 z_{1} z_{1}}\left[\left(Y_{1} \tilde{\pi}_{1}-K_{1} Y_{2} \tilde{\pi}_{2}\right)^{\top} \Sigma \Sigma^{\top}\left(Y_{1} \tilde{\pi}_{1}-K_{1} Y_{2} \tilde{\pi}_{2}\right)\right]+H_{1 v}[\kappa(\delta-v)] \\
\left.+\frac{1}{2} H_{1 v v} \sigma_{v}^{2} v+H_{1 z_{1} v}\left[\left(Y_{1} \tilde{\pi}_{1}-K_{1} Y_{2} \tilde{\pi}_{2}\right)^{\top} \Gamma\right]\right\}=0,
\end{array}
$$

with terminal condition

$$
\begin{aligned}
H_{1}\left(T, z_{1}, v\right) & =\sup _{\tilde{\pi}_{1}(t)} E\left[U_{1}\left(Z_{1}(T)\right) \mid Z_{1}(T)\right. \\
& \left.=z_{1}, V(T)=v\right]=U_{1}\left(z_{1}\right),
\end{aligned}
$$

where $\Gamma=\left(0, \sigma_{v} \rho v\right)^{\top}$.

Proof. On $[t, t+h]$, we choose any control process $\tilde{\pi}_{1}(t)$ and $\left(Z_{1}, V\right)$ evolves from $\left(t, Z_{1}(t), V(t)\right)$ to $\left(t+h, Z_{1}^{t, z_{1}, v}\left(t+h, \tilde{\pi}_{1}(t)\right), V(t+h)\right)$. According to It $\widehat{o}$ formula, we have

$$
\begin{aligned}
& H_{1}\left(t+h, Z_{1}(t+h), V(t+h)\right) \\
& =H_{1}\left(t, z_{1}, v\right)+\int_{t}^{t+h}\left(\frac{\partial H_{1}\left(u, Z_{1}(u), V(u)\right)}{\partial u}\right) \mathrm{d} u+\frac{\partial H_{1}\left(u, Z_{1}(u), V(u)\right)}{\partial Z_{1}} \mathrm{~d} Z_{1} \\
& \quad+\frac{1}{2} \frac{\partial^{2} H_{1}\left(u, Z_{1}(u), V(u)\right)}{\partial^{2} Z_{1}} \mathrm{~d}\left[Z_{1}, Z_{1}\right]_{u}+\frac{\partial H_{1}\left(u, Z_{1}(u), V(u)\right)}{\partial V} \mathrm{~d} V \\
& \left.+\frac{1}{2} \frac{\partial^{2} V_{1}\left(u, Z_{1}(u), V(u)\right)}{\partial^{2} V} \mathrm{~d}[V, V]_{u}+\frac{\partial^{2} H_{1}\left(u, Z_{1}(u), V(u)\right)}{\partial Z_{1} \partial V} \mathrm{~d}\left[Z_{1}, V\right]_{u}\right) \\
& =H_{1}\left(t, z_{1}, v\right)+\int_{t}^{t+h}\left[H_{1 u}\right]+H_{1 z_{1}}\left[\left(c_{1}-K_{1} c_{2}\right)+r Z_{1}(u)+\left[Y_{1} \tilde{\pi}_{i}-K_{1} Y_{2} \tilde{\pi}_{2}\right]^{\top} \Sigma \Lambda\right] \\
& \quad+\frac{1}{2} H_{1 z_{1} z_{1}}\left(\left(Y_{1} \tilde{\pi}_{1}-K_{1} Y_{2} \tilde{\pi}_{2}\right)^{\top} \Sigma \Sigma^{\top}\left(Y_{1} \tilde{\pi}_{1}-K_{1} Y_{2} \tilde{\pi}_{2}\right)\right)+H_{1 v}(\kappa(\delta-v)) \\
& \left.\quad+\frac{1}{2} H_{1 v v} \sigma_{v}^{2} \rho v+H_{1 z_{1}}\left(\left(Y_{1} \tilde{\pi}_{1}-K_{1} Y_{2} \tilde{\pi}_{2}\right)^{\top} \Gamma\right)\right] \mathrm{d} u \\
& +\int_{t}^{t+h} H_{1 z_{1}}\left[Y_{1} \tilde{\pi}_{i}-K_{1} Y_{2} \tilde{\pi}_{2}\right]^{\top} \Sigma \mathrm{d} W(u) .
\end{aligned}
$$


By dynamic programming principle, it follows that

$$
H_{1}\left(t, z_{1}, v\right)=\sup _{\tilde{\pi}_{1}(t)} E\left[H_{1}\left(t+h, Z_{1}(t+h), V(t+h)\right) \mid Z_{1}(t)=z_{1}, V(t)=v\right] .
$$

Then, inputting (20) into (21), we can obtain

$$
\begin{aligned}
0= & \sup _{\tilde{\pi}_{1}(t)} E\left[\int _ { t } ^ { t + h } \left[H_{1 u}+H_{1 z_{1}}\left[\left(c_{1}-K_{1} c_{2}\right)+r Z_{1}(u)+\left[Y_{1} \tilde{\pi}_{i}-K_{1} Y_{2} \tilde{\pi}_{2}\right]^{\top} \Sigma \Lambda\right]\right.\right. \\
& +\frac{1}{2} H_{1 z_{1} z_{1}}\left(\left(Y_{1} \tilde{\pi}_{1}-K_{1} Y_{2} \tilde{\pi}_{2}\right)^{\top} \Sigma \Sigma^{\top}\left(Y_{1} \tilde{\pi}_{1}-K_{1} Y_{2} \tilde{\pi}_{2}\right)\right)+H_{1 v}(\kappa(\delta-v)) \\
& \left.\left.+\frac{1}{2} H_{1 v v} \sigma_{v}^{2} \rho v+H_{1 z_{1} v}\left(\left(Y_{1} \tilde{\pi}_{1}-K_{1} Y_{2} \tilde{\pi}_{2}\right)^{\top} \Gamma\right)\right] \mid Z_{1}(t)=z_{1}, V(t)=v\right] .
\end{aligned}
$$

Note $\tilde{\pi}_{1}(t)$ in $(22)$ is a process on $[t, t+h]$, as $h \longrightarrow 0, \tilde{\pi}_{1}(u) \longrightarrow \tilde{\pi}_{1}(t)$. For equation (22), dividing by $h>0$ on its both sides and letting $h \longrightarrow 0$, by mean value theorem, as $u \longrightarrow t$, we have that $Z_{1}(u) \longrightarrow Z_{1}(t)=z_{1}$ and $V(u) \longrightarrow V(t)=v$. Thus, the following equality holds:

$$
\begin{aligned}
0= & \sup _{\tilde{\pi}_{1}(t)}\left\{H_{1 t}+H_{1 z_{1}}\left[\left(c_{1}-K_{1} c_{2}\right)+r z_{1}+\left(Y_{1} \tilde{\pi}_{1}-K_{1} Y_{2} \tilde{\pi}_{2}\right)^{\top} \Sigma \Lambda\right]\right. \\
& +\frac{1}{2} H_{1 z_{1} z_{1}}\left[\left(Y_{1} \tilde{\pi}_{1}-K_{1} Y_{2} \tilde{\pi}_{2}\right)^{\top} \Sigma \Sigma^{\top}\left(Y_{1} \tilde{\pi}_{1}-K_{1} Y_{2} \tilde{\pi}_{2}\right)\right]+H_{1 v}[\kappa(\delta-v)] \\
& \left.+\frac{1}{2} H_{1 v v} \sigma_{v}^{2} \rho v+H_{1 z_{1} v}\left[\left(Y_{1} \tilde{\pi}_{1}-K_{1} Y_{2} \tilde{\pi}_{2}\right)^{\top} \Gamma\right]\right\}=0 .
\end{aligned}
$$

Then, this theorem is proved.

Similar to Theorem 1, the HJB equation for Manager 2 can be presented by the following equality:

$$
\begin{aligned}
\sup _{\tilde{\pi}_{2}(t)}\left\{H_{2 t}\right. & +H_{2 z_{2}}\left[\left(c_{2}-K_{2} c_{1}\right)+r(t) Z_{2}+\left(Y_{2} \tilde{\pi}_{2}-K_{2} Y_{1} \tilde{\pi}_{1}\right)^{\top} \Sigma \Lambda\right] \\
& +\frac{1}{2} H_{2 z_{2} z_{2}}\left[\left(Y_{2} \tilde{\pi}_{2}-K_{2} Y_{1} \tilde{\pi}_{1}\right)^{\top} \Sigma \Sigma^{\top}\left(Y_{2} \tilde{\pi}_{2}-K_{2} Y_{1} \tilde{\pi}_{1}\right)\right]+H_{2 v}[\kappa(\delta-v)] \\
& \left.+\frac{1}{2} H_{2 v v} \sigma_{v}^{2} \rho v+H_{2 z_{2} v}\left[\left(Y_{2} \tilde{\pi}_{2}-K_{2} Y_{1} \tilde{\pi}_{1}\right)^{\top} \Gamma\right]\right\}=0,
\end{aligned}
$$

with terminal condition

$$
H_{2}\left(T, z_{2}, v\right)=\sup _{\tilde{\pi}_{2}(t)} E\left[U_{2}\left(Z_{2}(T)\right) \mid Z_{2}(T)=z_{2}, V(T)=v\right]=U_{2}\left(z_{2}\right)
$$


where $\Gamma=\left(0, \sigma_{\nu} \rho v\right)^{\top}$.

Based on the HJB equations (18) and (24) above, the optimal investment strategy of the two managers can be achieved.
Theorem 2. The Nash equilibrium strategies $\left(\pi_{1}^{*}, \pi_{2}^{*}\right)$ for the relative performance concerning DC pension managers are as follows:

$$
\begin{aligned}
& \pi_{1}^{*}(t)=\frac{\left(K_{1} /\left(\gamma_{2}+k_{2}\left(\gamma_{1}-\gamma_{2}\right)\right)+1 /\left(\gamma_{1}+k_{1}\left(\gamma_{2}-\gamma_{1}\right)\right)\right)\left[\left(\Sigma^{\top}\right)^{-1} \Lambda-g(t)\left(\Sigma^{\top}\right)^{-1} \Sigma^{-1} \Gamma\right]}{\left(1-K_{1} K_{2}\right) Y_{1} a(t)}+(1,0)^{\top}, \\
& \pi_{2}^{*}(t)=\frac{\left(K_{2} /\left(\gamma_{1}+k_{1}\left(\gamma_{2}-\gamma_{1}\right)\right)+1 /\left(\gamma_{2}+k_{2}\left(\gamma_{1}-\gamma_{2}\right)\right)\right)\left[\left(\Sigma^{\top}\right)^{-1} \Lambda-g(t)\left(\Sigma^{\top}\right)^{-1} \Sigma^{-1} \Gamma\right]}{\left(1-K_{1} K_{2}\right) Y_{2} a(t)}+(1,0)^{\top}, \\
& \tilde{\pi}_{1}^{*}=\frac{K_{1} Y_{2} \widetilde{\pi}_{2}}{Y_{1}}-\frac{H_{1 z_{1}}}{H_{1 z_{1} z_{1}} Y_{1}}\left(\Sigma^{\top}\right)^{-1} \Lambda-\frac{H_{1 z_{1} v}}{H_{1 z_{1} z_{1}} Y_{1}}\left(\Sigma^{\top}\right)^{-1} \Sigma^{-1} \Gamma .
\end{aligned}
$$

where

$$
\begin{aligned}
& a(t)=e^{r(T-t)}, \\
& g(t)=\frac{\lambda_{1} \lambda_{2}\left[e^{A\left(\lambda_{1}-\lambda_{2}\right)(T-t)}-1\right]}{\lambda_{1} e^{A\left(\lambda_{1}-\lambda_{2}\right)(T-t)}-\lambda_{2}},
\end{aligned}
$$

and $\lambda_{1,2}=\left(-\left(k+\lambda_{s} \sigma_{v} \rho\right) \pm \sqrt{\kappa^{2}+2 \kappa \lambda_{s} \sigma_{v} \rho+\sigma_{v}^{2} \lambda_{s}^{2}}\right) /\left(\sigma_{v}^{2}(1-\right.$ $\left.\left.\rho^{2}\right)\right), A=(1 / 2) \sigma_{v}^{2}\left(1-\rho^{2}\right)$.

Similarly, according to the HJB equation (24), the form of $\tilde{\pi}_{2}^{*}$ has the form as

$$
\tilde{\pi}_{2}^{*}=\frac{K_{2} Y_{1} \widetilde{\pi}_{1}}{Y_{2}}-\frac{H_{2 z_{2}}}{H_{2 z_{2} z_{2}} Y_{2}}\left(\Sigma^{\top}\right)^{-1} \Lambda-\frac{H_{2 z_{2} v}}{H_{2 z_{2} z_{2}} Y_{2}}\left(\Sigma^{\top}\right)^{-1} \Sigma^{-1} \Gamma \text {. }
$$

Proof. According to the HJB equation (18), using the first order condition, we have that

Substitute $\widetilde{\pi}_{2}^{*}$ into (28) and $\tilde{\pi}_{1}^{*}$ into (29), respectively; then, (28) and (29) are reduced to be

$$
\begin{aligned}
& \tilde{\pi}_{1}^{*}=-\frac{1}{\left(1-K_{1} K_{2}\right) Y_{1}}\left\{\left[\frac{K_{1} H_{2 z_{2}}}{H_{2 z_{2} z_{2}}}+\frac{H_{1 z_{1}}}{H_{1 z_{1} z_{1}}}\right]\left(\Sigma^{\top}\right)^{-1} \Lambda+\left[\frac{K_{1} H_{2 z_{2} v}}{H_{2 z_{2} z_{2}}}+\frac{H_{1 z_{1} v}}{H_{1 z_{1} z_{1}}}\right]\left(\Sigma^{\top}\right)^{-1} \Sigma^{-1} \Gamma\right\}, \\
& \tilde{\pi}_{2}^{*}=-\frac{1}{\left(1-K_{1} K_{2}\right) Y_{2}}\left\{\left[\frac{K_{2} H_{1 z_{1}}}{H_{1 z_{1} z_{1}}}+\frac{H_{2 z_{2}}}{H_{2 z_{2} z_{2}}}\right]\left(\Sigma^{\top}\right)^{-1} \Lambda+\left[\frac{K_{2} H_{1 z_{1} v}}{H_{1 z_{1} z_{1}}}+\frac{H_{2 z_{2} v}}{H_{2 z_{2} z_{2}}}\right]\left(\Sigma^{\top}\right)^{-1} \Sigma^{-1} \Gamma\right\} .
\end{aligned}
$$

We conjecture that the optimal utility function for manager $i$ is as follows:

$$
H_{i}\left(t, z_{i}, v\right)=-\frac{1}{\widetilde{\gamma}_{i}} e^{-\tilde{\gamma}_{i}\left[a_{i}(t)\left(z_{i}-b_{i}(t)\right)+g_{i}(t) v\right]},
$$

where $\tilde{\gamma}_{1}=\gamma_{1}+k_{1}\left(\gamma_{2}-\gamma_{1}\right)$ and $\tilde{\gamma}_{2}=\gamma_{2}+k_{2}\left(\gamma_{1}-\gamma_{2}\right)$. For the boundary condition, we have $a_{i}(T)=1, b_{i}(T)=0$, and $g_{i}(T)=0$.

Substitute $H_{1}, H_{2}, \widetilde{\pi}_{1}^{*}$, and $\tilde{\pi}_{2}^{*}$ into HJB equations (18) and (24), and for simplicity, let $\Delta_{1}=c_{1}-K_{1} c_{2}$ and $\Delta_{2}=c_{2}-K_{2} c_{1}$; then, the following equality holds:

$$
\begin{aligned}
& a_{i}^{\prime}(t)\left(z_{i}(t)-b_{i}(t)\right)-b_{i}^{\prime}(t) a_{i}(t)+g_{i}^{\prime}(t) v+a_{i}(t)\left[\Delta_{i}+r z_{i}-\frac{g_{i}(t)}{a_{i}(t)} \Gamma^{\top}\left(\Sigma^{-1}\right)^{\top} \Lambda\right. \\
& \left.+\frac{\Lambda^{\top} \Lambda}{\tilde{\gamma}_{i} a_{i}(t)}\right]-\frac{\widetilde{\gamma}_{i} a_{i}^{2}(t)}{2}\left[\frac{g_{i}^{2}(t)}{a_{i}^{2}(t)} \Gamma^{\top}\left(\Sigma^{-1}\right)^{\top} \Sigma^{-1} \Gamma-\frac{g_{i}(t)}{\tilde{\gamma}_{i} a_{i}^{2}(t)} \Gamma^{\top}\left(\Sigma^{-1}\right)^{\top} \Lambda-\frac{g_{i}(t)}{\tilde{\gamma}_{i} a_{i}^{2}(t)} \Lambda^{\top} \Sigma^{-1} \Gamma\right. \\
& \left.\quad+\frac{1}{\tilde{\gamma}_{i}^{2} a_{i}^{2}(t)} \Lambda^{\top} \Lambda\right]+\kappa(\delta-v) g_{i}(t)-\frac{1}{2} \widetilde{\gamma}_{i} \sigma_{v}^{2} v g_{i}^{2}(t)+\tilde{\gamma}_{i} a_{i}(t) g_{i}(t)\left[\frac{g_{i}(t)}{a_{i}(t)} \Gamma^{\top}\left(\Sigma^{-1}\right)^{\top} \Sigma^{-1} \Gamma\right. \\
& \left.\quad-\frac{1}{\tilde{\gamma}_{i} a_{i}(t)} \Lambda^{\top} \Sigma^{-1} \Gamma\right]=0 .
\end{aligned}
$$


After some reductions for (33), we obtain that

$$
\begin{aligned}
& \left(a_{i}^{\prime}(t)+r a_{i}(t)\right) z_{i}+\left[-a_{i}^{\prime}(t) b_{i}(t)-a_{i}(t) b_{1}^{\prime}(t)+a_{i}(t) \Delta_{i}+g_{i}(t) \kappa \delta\right] \\
& +g_{i}^{\prime}(t) v-g_{i}(t) \Gamma^{\top}\left(\Sigma^{-1}\right)^{\top} \Lambda+\frac{1}{\widetilde{\gamma}_{i}} \Lambda^{\top} \Lambda-\frac{1}{2} \widetilde{\gamma}_{i} g_{i}^{2}(t) \Gamma^{\top}\left(\Sigma^{-1}\right)^{\top} \Sigma^{-1} \Gamma \\
& \quad+\frac{1}{2} g_{i}(t) \Gamma^{\top}\left(\Sigma^{-1}\right)^{\top} \Lambda+\frac{1}{2} g_{i}(t) \Lambda^{\top} \Sigma^{-1} \Gamma-\frac{1}{2} \widetilde{\gamma}_{i}^{\top} \Lambda-g_{i}(t) \kappa v-\frac{1}{2} \widetilde{\gamma}_{i} g_{i}^{2}(t) \sigma_{v}^{2} V \\
& +\widetilde{\gamma}_{i} g_{i}^{2}(t) \Gamma^{\top}\left(\Sigma^{-1}\right)^{\top} \Sigma^{-1} \Gamma-g_{i}(t) \Lambda^{\top} \Sigma^{-1} \Gamma=0 .
\end{aligned}
$$

Comparing the coefficient of $z_{i}$ and $v$, we can get three ordinary differential equations as follows:

$$
\begin{aligned}
& \left\{\begin{array}{l}
a_{i}^{\prime}(t)+r a_{i}(t)=0, \\
a_{i}(T)=1,
\end{array}\right. \\
& \left\{\begin{array}{l}
-a_{i}^{\prime}(t) b_{i}(t)-a_{i}(t) b_{i}^{\prime}(t)+a_{i}(t) \Delta_{i}+g_{i}(t) \kappa \delta+\frac{\left(\lambda_{p}-\sigma_{p}\right)^{2}}{2 \widetilde{\gamma}_{i}}=0, \\
b_{i}(T)=0,
\end{array}\right. \\
& \left\{\begin{array}{l}
g_{i}^{\prime}(t)-g_{i}(t) \sigma_{v} \lambda_{s} \rho+\frac{1}{2 \widetilde{\gamma}_{i}} \lambda_{s}^{2}-\frac{1}{2} \widetilde{\gamma}_{i} g_{i}^{2}(t) \sigma_{v}^{2}\left(1-\rho^{2}\right)-g_{i}(t) \kappa=0, \\
g_{i}(T)=0 .
\end{array}\right.
\end{aligned}
$$

Solving these three equations in (35)-(37), we can get the solutions of $a_{i}(t), b_{i}(t)$, and $g_{i}(t)$ as

$$
\begin{aligned}
a_{i}(t)= & e^{r(T-t)} \\
b_{i}(t)= & \frac{\Delta_{i}}{r}\left[e^{-r(T-t)}-1\right]-\frac{\left(\lambda_{p}-\sigma_{p}\right)^{2}}{2 \widetilde{\gamma}_{i}}(T-t) e^{-r(T-t)}-\frac{\kappa \delta \lambda_{2}(T-t)}{\widetilde{\gamma}_{i}} e^{-r(T-t)} \\
& +\frac{\kappa \delta}{A \widetilde{\gamma}_{i}} e^{-r(T-t)}\left[\ln \left(1-\frac{\lambda_{2}}{\lambda_{1}}\right)-\ln \left(1-\frac{\lambda_{2}}{\lambda_{1}} e^{-A\left(\lambda_{1}-\lambda_{2}\right)(T-t)}\right)\right], \\
g_{i}(t)= & \frac{1}{\widetilde{\gamma}_{i}} \frac{\lambda_{1} \lambda_{2}\left[e^{A\left(\lambda_{1}-\lambda_{2}\right)(T-t)}-1\right]}{\lambda_{1} e^{A\left(\lambda_{1}-\lambda_{2}\right)(T-t)}-\lambda_{2}}
\end{aligned}
$$

$$
\begin{array}{lcccc}
\text { where } A=(1 / 2) \sigma_{v}^{2}\left(1-\rho^{2}\right) & \text { and } & \text { Let } & a(t)=a_{1}(t)=a_{2}(t) & \text { and } \\
\lambda_{1,2}=\left(-\left(k+\lambda_{s} \sigma_{v} \rho\right) \pm \sqrt{\kappa^{2}+2 \kappa \lambda_{s} \sigma_{v} \rho+\sigma_{v}^{2} \lambda_{s}^{2}}\right) /\left(\sigma_{v}^{2}\left(1-\rho^{2}\right)\right) . & g(t)=\left(\lambda_{1} \lambda_{2}\left[e^{A\left(\lambda_{1}-\lambda_{2}\right)(T-t)}-1\right]\right) /\left(\lambda_{1} e^{A\left(\lambda_{1}-\lambda_{2}\right)(T-t)}-\lambda_{2}\right) .
\end{array}
$$


Thus, we can get $H_{i}\left(t, z_{i}, v\right)$. Putting $H_{i}\left(t, z_{i}, v\right)$ in (30) and (31), respectively, we have that

$$
\begin{aligned}
& \tilde{\pi}_{1}^{*}(t)=\frac{\left(K_{1} /\left(\gamma_{2}+k_{2}\left(\gamma_{1}-\gamma_{2}\right)\right)+1 /\left(\gamma_{1}+k_{1}\left(\gamma_{2}-\gamma_{1}\right)\right)\right)\left[\left(\Sigma^{\top}\right)^{-1} \Lambda-g(t)\left(\Sigma^{\top}\right)^{-1} \Sigma^{-1} \Gamma\right]}{\left(1-K_{1} K_{2}\right) Y_{1} a(t)}, \\
& \tilde{\pi}_{2}^{*}(t)=\frac{\left(K_{2} /\left(\gamma_{1}+k_{1}\left(\gamma_{2}-\gamma_{1}\right)\right)+1 /\left(\gamma_{2}+k_{2}\left(\gamma_{1}-\gamma_{2}\right)\right)\right)\left[\left(\Sigma^{\top}\right)^{-1} \Lambda-g(t)\left(\Sigma^{\top}\right)^{-1} \Sigma^{-1} \Gamma\right]}{\left(1-K_{1} K_{2}\right) Y_{2} a(t)} .
\end{aligned}
$$

Since we have $\tilde{\pi}_{i}(t)=\left(\pi_{p i}(t), \pi_{s i}(t)\right)^{\top}-(1,0)^{\top}$, then we get the conclusion, which proved the theorem.
Remark 3. More specifically, we have the optimal strategies of IIB and stock as follows:

Remark 2. After some simple calculation, we can get that $a(t) \geq 0, g(t) \leq 0$ and $a(t), g(t)$ have no relationship with $\gamma_{i}$, $K_{i}, k_{i}(i=1,2)$.

$$
\begin{aligned}
& \pi_{1 I}^{*}(t)=\left[\frac{K_{1}}{\gamma_{2}+k_{2}\left(\gamma_{1}-\gamma_{2}\right)}+\frac{1}{\gamma_{1}+k_{1}\left(\gamma_{2}-\gamma_{1}\right)}\right] \frac{\lambda_{p}-\sigma_{p}-\sigma_{s} \lambda_{s}+\sigma_{s} \sigma_{v} \rho g(t)}{\sigma_{p}\left(1-K_{1} K_{2}\right) Y_{1} a(t)}+1, \\
& \pi_{1 S}^{*}(t)=\left[\frac{K_{1}}{\gamma_{2}+k_{2}\left(\gamma_{1}-\gamma_{2}\right)}+\frac{1}{\gamma_{1}+k_{1}\left(\gamma_{2}-\gamma_{1}\right)}\right] \frac{\lambda_{s}-\sigma_{v} \rho g(t)}{\left(1-K_{1} K_{2}\right) Y_{1} a(t)}, \\
& \pi_{2 I}^{*}(t)=\left[\frac{K_{2}}{\gamma_{1}+k_{1}\left(\gamma_{2}-\gamma_{1}\right)}+\frac{1}{\gamma_{2}+k_{2}\left(\gamma_{1}-\gamma_{2}\right)}\right] \frac{\lambda_{p}-\sigma_{p}-\sigma_{s} \lambda_{s}+\sigma_{s} \sigma_{v} \rho g(t)}{\sigma_{p}\left(1-K_{1} K_{2}\right) Y_{2} a(t)}+1, \\
& \pi_{2 S}^{*}(t)=\left[\frac{K_{2}}{\gamma_{1}+k_{1}\left(\gamma_{2}-\gamma_{1}\right)}+\frac{1}{\gamma_{2}+k_{2}\left(\gamma_{1}-\gamma_{2}\right)}\right] \frac{\lambda_{s}-\sigma_{v} \rho g(t)}{\left(1-K_{1} K_{2}\right) Y_{2} a(t)} .
\end{aligned}
$$

In the following, we try to compare the optimal investment strategy of RPC manager with traditional Merton manager (here, we mean managers who have no relative performance concern with other managers, i.e., we consider the optimal strategy with one manager only). Moreover, we compared the optimal strategy between RRAC manager and RWC manager and obtained some interesting results.

Corollary 1. The traditional Merton optimal investment strategy is as follows:

$$
\pi_{M}^{*}(t)=\frac{1}{\gamma Y a(t)}\left[\left(\Sigma^{\top}\right)^{-1} \Lambda-g(t)\left(\Sigma^{\top}\right)^{-1} \Sigma^{-1} \Gamma\right]+(1,0)^{\top},
$$

where $a(t)$ and $g(t)$ are as the ones in (27).

Proof. Let $K_{i}=0$ and $k_{i}=0(i=1,2)$ in Theorem 2; then, we can obtain the conclusion.
Remark 4. More specifically, we can get the optimal investment strategy of Merton manager on IIB and stock as follows:

$$
\begin{aligned}
& \pi_{\mathrm{MI}}^{*}(t)=\frac{1}{\gamma Y a(t)} \frac{\lambda_{p}-\sigma_{p}-\sigma_{s} \lambda_{s}+\sigma_{s} \sigma_{v} \rho g(t)}{\sigma_{p}}+1, \\
& \pi_{\mathrm{MS}}^{*}(t)=\frac{1}{\gamma Y a(t)}\left(\lambda_{s}-\sigma_{v} \rho g(t)\right) .
\end{aligned}
$$

We consider the situation that the manager has only relative wealth concern, which means that the manager is only concerned about another manager's wealth. As mentioned before, the existing literature mainly focuses on the optimal strategies of managers with relative wealth concern. Using Theorem 2, we get the optimal strategy of relative wealth concern manager under inflation risk. Meanwhile, we compare the difference between traditional Merton optimal strategy with the case of RWC. Then, we have the following results. 
Corollary 2. The optimal portfolio strategy of the pension manager who has relative wealth concern only is as follows:

$$
\begin{aligned}
& \pi_{1}^{*}(t)=\frac{\left(\left(K_{1} / \gamma_{2}\right)+\left(1 / \gamma_{1}\right)\right)\left[\left(\Sigma^{\top}\right)^{-1} \Lambda-g(t)\left(\Sigma^{\top}\right)^{-1} \Sigma^{-1} \Gamma\right]}{\left(1-K_{1} K_{2}\right) Y_{1} a(t)}+(1,0)^{\top}, \\
& \pi_{2}^{*}(t)=\frac{\left(\left(K_{2} / \gamma_{1}\right)+\left(1 / \gamma_{2}\right)\right)\left[\left(\Sigma^{\top}\right)^{-1} \Lambda-g(t)\left(\Sigma^{\top}\right)^{-1} \Sigma^{-1} \Gamma\right]}{\left(1-K_{1} K_{2}\right) Y_{2} a(t)}+(1,0)^{\top} .
\end{aligned}
$$

Proof. Let $k_{i}=0, i=1,2$, in Theorem 2; then, we can get the conclusion.

Remark 5. The optimal strategy with the RWC managers on IIB and stock is as follows:

$$
\begin{aligned}
& \pi_{1 I}^{*}(t)=\left(\frac{K_{1}}{\gamma_{2}}+\frac{1}{\gamma_{1}}\right) \frac{\lambda_{p}-\sigma_{p}-\sigma_{s} \lambda_{s}+\sigma_{s} \sigma_{v} \rho g(t)}{\sigma_{p}\left(1-K_{1} K_{2}\right) Y_{1} a(t)}+1 \\
& \pi_{1 S}^{*}(t)=\left(\frac{K_{1}}{\gamma_{2}}+\frac{1}{\gamma_{1}}\right) \frac{\left(\lambda_{s}-\sigma_{v} \rho g(t)\right)}{\left(1-K_{1} K_{2}\right) Y_{1} a(t)} \\
& \pi_{2 I}^{*}(t)=\left(\frac{K_{2}}{\gamma_{1}}+\frac{1}{\gamma_{2}}\right) \frac{\left(\lambda_{p}-\sigma_{p}-\sigma_{s} \lambda_{s}+\sigma_{s} \sigma_{v} \rho g(t)\right)}{\sigma_{p}\left(1-K_{1} K_{2}\right) Y_{2} a(t)}+1, \\
& \pi_{2 S}^{*}(t)=\left(\frac{K_{2}}{\gamma_{1}}+\frac{1}{\gamma_{2}}\right) \frac{\left(\lambda_{s}-\sigma_{v} \rho g(t)\right)}{\left(1-K_{1} K_{2}\right) Y_{2} a(t)}
\end{aligned}
$$

Comparing (46) and (48) with (43), we can find that being relative wealth concern, both manager 1 and manager 2 will put more proportion of wealth on stock (long/short position) than that of traditional Merton optimal strategy, but the proportion on IIB is not clear. Moreover, as in Corollary 1 , relative wealth concern is also in some way equivalent to changing Merton managers' risk aversion coefficient.

We further consider another special situation. That is, the manager only cares about the risk attitude of the other manager. In fact, through the comparison of the optimal investment strategies of RPC, RWC manager with Merton manager, we find that the optimal strategy between two competing managers can be explained only as a change in the risk aversion coefficient. In other words, relative performance concern or relative wealth concern has distorted the risk aversion coefficient of the Merton manager. Therefore, we further consider the optimal investment strategy for managers who simply consider the risk aversion coefficient of the other manager, and we get the conclusion as follows.
Corollary 3. The optimal strategy when the managers only have risk aversion concern is given as follows:

$$
\begin{aligned}
& \pi_{1}^{*}(t)=\frac{\left.\left(\Sigma^{\top}\right)^{-1} \Lambda-g(t)\left(\Sigma^{\top}\right)^{-1} \Sigma^{-1} \Gamma\right]}{\left(\gamma_{1}+k_{1}\left(\gamma_{2}-\gamma_{1}\right) Y_{1} a(t)\right)}+(1,0)^{\top}, \\
& \pi_{2}^{*}(t)=\frac{\left[\left(\Sigma^{\top}\right)^{-1} \Lambda-g(t)\left(\Sigma^{\top}\right)^{-1} \Sigma^{-1} \Gamma\right]}{\left(\gamma_{2}+k_{2}\left(\gamma_{1}-\gamma_{2}\right)\right) Y_{2} a(t)}+(1,0)^{\top} .
\end{aligned}
$$

Proof. Let $K_{i}=0, i=1,2$, in Theorem 2, then we can get the conclusion.

Remark 6. The optimal strategy with the RRAC managers on IIB and stock is as follows:

$$
\begin{aligned}
& \pi_{I 1}^{*}(t)=\frac{\lambda_{p}-\sigma_{p}-\sigma_{s} \lambda_{s}+\sigma_{s} \sigma_{v} \rho g(t)}{\left(\gamma_{1}+k_{1}\left(\gamma_{2}-\gamma_{1}\right)\right) Y_{1} a(t) \sigma_{p}}+1, \\
& \pi_{S 1}^{*}(t)=\frac{\lambda_{s}-\sigma_{v} \rho g(t)}{\left(\gamma_{1}+k_{1}\left(\gamma_{2}-\gamma_{1}\right)\right) Y_{1} a(t)}, \\
& \pi_{I 2}^{*}(t)=\frac{\lambda_{p}-\sigma_{p}-\sigma_{s} \lambda_{s}+\sigma_{s} \sigma_{v} \rho g(t)}{\left(\gamma_{2}+k_{2}\left(\gamma_{1}-\gamma_{2}\right)\right) Y_{2} a(t) \sigma_{p}}+1, \\
& \pi_{S 2}^{*}(t)=\frac{\lambda_{s}-\sigma_{v} \rho g(t)}{\left(\gamma_{2}+k_{2}\left(\gamma_{1}-\gamma_{2}\right)\right) Y_{2} a(t)} .
\end{aligned}
$$

3.3. A Special Case. In the rest of this section, we will consider a special case that manager 1 is a normal pension manager in the pension market, which means that he/she cannot influence the market. The other manager (manager 2) is a representative pension manager, who has average risk aversion and average wealth. Under this assumption, it follows that $K_{2}=k_{2}=0$ (i.e., manager 2 will not compete with manager 1 , but manager 1 will try to compete with manager 2). Then, according to Corollary 2, the optimal strategy of manager 1 while he/she is a RWC manager is as follows:

$$
\pi_{1}^{*}(t)=\frac{\left(\left(K_{1} / \gamma_{2}\right)+\left(1 / \gamma_{1}\right)\right)\left[\left(\Sigma^{\top}\right)^{-1} \Lambda-g(t)\left(\Sigma^{\top}\right)^{-1} \Sigma^{-1} \Gamma\right]}{Y_{1} a(t)}+(1,0)^{\top} .
$$

We compare this formula with the situation, in which manager 1 is a RRAC manager. Then, according to Corollary 3 , the optimal strategy is as follows:

$$
\pi_{1}^{*}(t)=\frac{\left.\left(\Sigma^{\top}\right)^{-1} \Lambda-g(t)\left(\Sigma^{\top}\right)^{-1} \Sigma^{-1} \Gamma\right]}{\left(\gamma_{1}+k_{1}\left(\gamma_{2}-\gamma_{1}\right) Y_{1} a(t)\right)}+(1,0)^{\top} .
$$

Comparing (51) with (52), we can find they have similar forms. Especially, if we let $K_{1}=k_{1}$, which means that manager 1 will take the same weight of RWC and RRAC in optimal strategy. As previously analyzed, both relative risk 
aversion concern and relative wealth concern are essentially a distortion of the manager's risk aversion coefficient in Merton's optimization strategy. Comparing (51) and (52) with traditional Merton optimal strategy (41), when manager 1 is a RWC manager, then the optimal investment strategy in (51) can be regarded as a Merton manager's optimal strategy with risk aversion coefficient $1 /\left(\left(K_{1} / \gamma_{2}\right)+\left(1 / \gamma_{1}\right)\right)$. Similarly, the optimal investment strategy of manager 1 when he/she is a RRAC manager in (52) can be regarded as a Merton manager's optimal strategy with risk aversion coefficient $\gamma_{1}+k_{1}\left(\gamma_{2}-\gamma_{1}\right)$. Then, after some simple calculations, we have the following inequalities:

$$
\begin{aligned}
& \frac{1}{\left(K_{1} / \gamma_{2}\right)+\left(1 / \gamma_{1}\right)} \leq \gamma_{1}+k_{1}\left(\gamma_{2}-\gamma_{1}\right), \\
& \frac{1}{\left(K_{1} / \gamma_{2}\right)+\left(1 / \gamma_{1}\right)} \leq \gamma_{1},
\end{aligned}
$$

which means RRAC and Merton manager in fact have a higher risk aversion coefficient than that of RWC manger, but the relationship between the RRAC manager and Merton manager depends on the relationship between $\gamma_{1}$ and $\gamma_{2}$.

\section{Numerical Analysis}

This section illustrates several numerical examples to support our optimal investment strategy. To better understand the influence of the parameters on the optimal investment strategy of pension managers, we observe the ratio changes in investment strategies for stocks and IIB as pension managers become more competitive. Meanwhile, we also compare and analyze the optimal strategies of different type (RPC, RWC, RRAC, and Merton) manager in stock and IIB. The employed parameters of the model are based on the following annualized benchmark parameter values presented in Table 1, which exactly is the same as in [18].

Figure 1 compares and analyzes optimal investment strategies of manager 1 on stock as a RPC, RWC, RRAC, and Merton manager. In the parameter setting, we assume that manager 1 is more risk preference than manager 2. Under this assumption, from the graph, we can see that when manager 1 is an RRAC manager, his/her investment proportion on stock is the lowest. This is mainly because manager 1 is affected by manager 2 in risk appetite, increasing his/her risk aversion coefficient, which leads to reduce the investment proportion on stock. When manager 1 is a RWC manager, his/her relative investment share on stock is the highest, which is in line with our previous conclusion: the manager of RWC type is always more inclined to invest more shares on the stock than those of the Merton type. The proportion of RPC-type is lower than that of RWC-type, which is mainly affected by the risk aversion of investor 2. Compared with the RWC manager, the proportion of stock on RPC manager is reduced, but it is still larger than the Merton manager.

Figure 2 discusses the optimal investment strategy for stock when manager 2 is a RPC, RWC, RRAC, and Merton manager. We find that because manager 2 is more risk averse than manager 1 , it shows a somewhat opposite investment tendency on the stock optimal investment strategy. The classic Merton optimal investment strategy shows that manager 2 will invest a lower proportion in stock assets, while RRAC will make manager 2 invest a higher proportion than Merton optimal strategy in stock because manager 2 is subject to the lower risk aversion preference of manager 1, which reduces his/her level of risk aversion. At the same time, according to our previous conclusions, RWC investors tend to invest in a higher proportion of assets in stocks than the Merton manager. The RPC investor can be regarded as an investment strategy that further reduces the risk aversion based on the RWC manager.

Figures 3 and 4 describe the changes in the proportion of RPC, RWC, RRAC, and Merton manager investing on IIB over time. We find that different types of manager perform the opposite on IIB as they do on stock. For example, in Figure 3, the RWC manager has the lowest investment proportion on IIB. Correspondingly, among the several types of investors in Figure 1, he/she has adopted the highest proportion of investment on stock. At the same time, contrary to Figure 1, the investment proportion of several types of managers on IIB is decreasing with respect to time horizon.

Figure 5 shows the influence of the preference of manager 1 on relative wealth concern. From this figure, we find that if a manager pays more attention on his relative wealth than his own wealth, which means the greater the value of $K_{1}$ is, the easier the pension manager has a more increasing tendency to invest stock. In Figure 5, for $K_{1}=0.75$, the investment strategy of manager 1 on stock is far more aggressive than that $K_{1}=0.25$. This shows that, in order to exceed the competitor with the relative wealth, the manager can easily adopt a more aggressive investment strategy and increase the proportion of stock as time passes by.

In Figure 6, we consider the impact of the emphasis for RWC of manager 2 on his stock investment strategy. It reflects that pension manager 2 has a similar strategy as manager 1 . That is, the higher the weight on relative wealth is, easier it will be for pension managers to adopt aggressive investment strategies on stock. However, comparing with pension manager 1, pension manager 2 is more risk averse. Although manager 2 also increases the position of stock with the increase of the relative wealth weight, we can see that manager 2 is much more moderate compared with Figure 5. It means that the position of stock is much lower than that of manager 1 .

From Figures 5 and 6, the evolution of the Nash equilibrium strategy over time can be seen clearly. As long 
TABLE 1: Model parameters.

\begin{tabular}{|c|c|c|c|c|c|c|c|c|c|c|c|c|c|c|}
\hline$\gamma_{1}$ & $\gamma_{2}$ & $K_{1}$ & $K_{2}$ & $k_{1}$ & $k_{2}$ & $r$ & $\lambda_{P}$ & $\lambda_{S}$ & $\sigma_{P}$ & $\sigma_{S}$ & $\kappa$ & $\sigma_{V}$ & $t$ & $T$ \\
\hline 2 & 3 & 0.25 & 0.25 & 0.25 & 0.25 & 0.02 & 0.015 & 0.6 & 0.2 & 0.5 & 0.2 & 0.1 & 0 & 10 \\
\hline
\end{tabular}
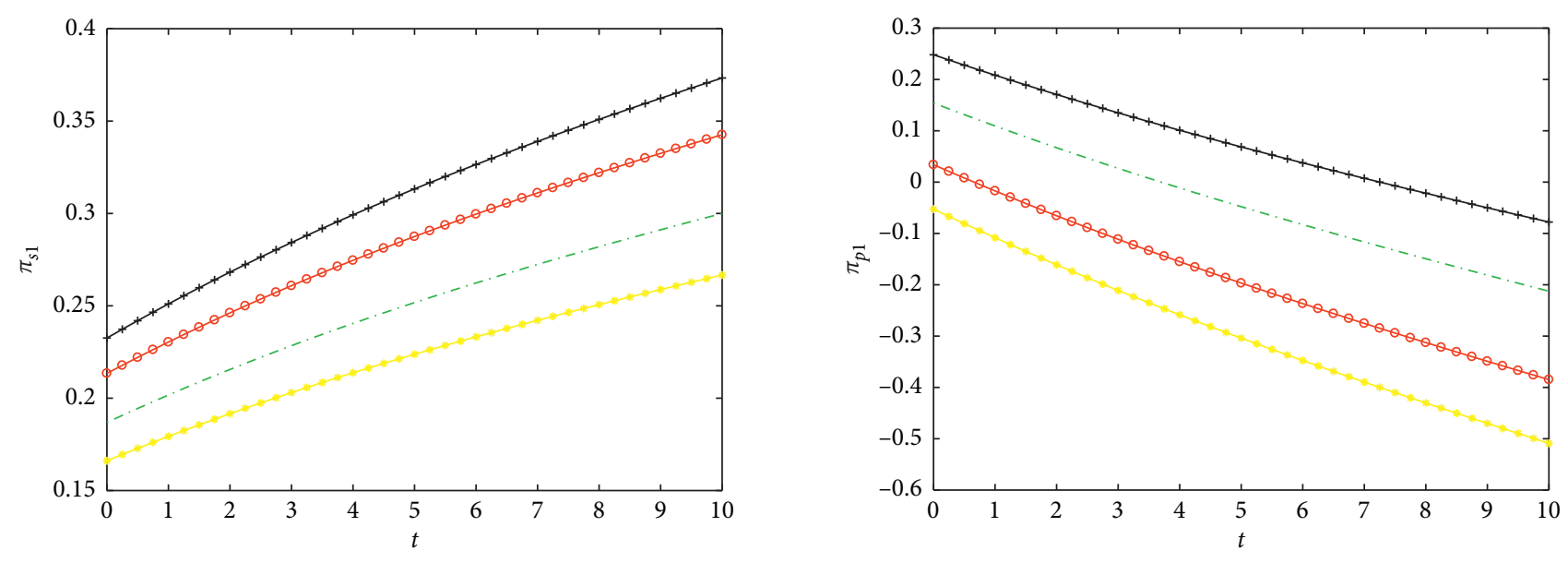

$$
\begin{aligned}
& \multimap \text { RPC: } K_{1}=K_{2}=k_{1}=k_{2}=0.25 \\
& \longrightarrow \text { RWC: } K_{1}=K_{2}=0.25, k_{1}=k_{2}=0 \\
& \longrightarrow \text { RRAC: } K_{1}=K_{2}=0, k_{1}=k_{2}=0.25 \\
& \cdots \text { Merton: } K_{1}=K_{2}=k_{1}=k_{2}=0
\end{aligned}
$$

FIGURE 1: Optimal investment strategy on stocks for manager 1 under RPC, RRAC, RWC, and Merton optimal strategy versus time $t$.

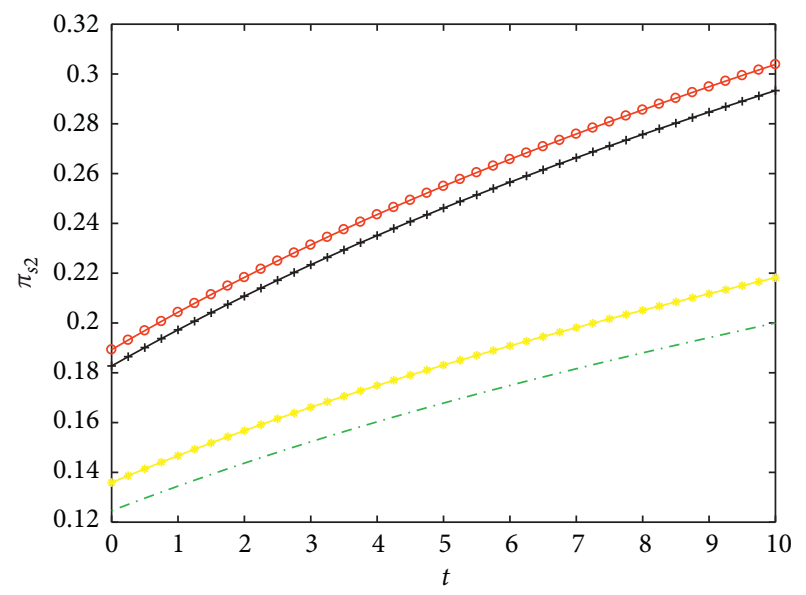

$$
\begin{aligned}
& \rightarrow \text { RPC: } K_{1}=K_{2}=k_{1}=k_{2}=0.25 \\
& \text { — RWC: } K_{1}=K_{2}=0.25, k_{1}=k_{2}=0 \\
& \text { RRAC: } K_{1}=K_{2}=0, k_{1}=k_{2}=0.25 \\
& -- \text { Merton: } K_{1}=K_{2}=k_{1}=k_{2}=0
\end{aligned}
$$

FIGURE 2: Optimal investment strategy on stocks for manager 2 under RPC, RRAC, RWC, and Merton optimal strategy versus time $t$.

as the original equilibrium of one party breaks and they increase the proportion of their asset on stock, the other manager will also follow-up to increase the proportion of stock in order to achieve a new balance. Furthermore, we see that manager 1 and manager 2 increasingly invest their assets on stock during the entire investment period in order

$$
\begin{aligned}
& \rightarrow \text { RPC: } K_{1}=K_{2}=k_{1}=k_{2}=0.25 \\
& \rightarrow \text { RWC: } K_{1}=K_{2}=0.25, k_{1}=k_{2}=0 \\
& \rightarrow \text { RRAC: } K_{1}=K_{2}=0, k_{1}=k_{2}=0.25 \\
& -- \text { Merton: } K_{1}=K_{2}=k_{1}=k_{2}=0
\end{aligned}
$$

FIgURE 3: Optimal investment strategy on IIB for manager 1 under RPC, RRAC, RWC, and Merton optimal strategy versus time $t$.

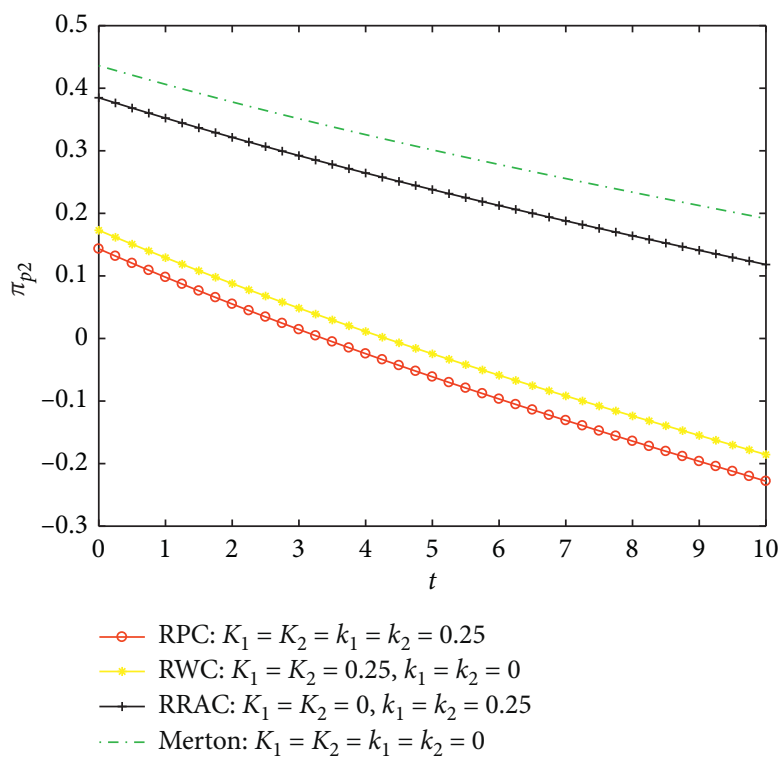

FIGURE 4: Optimal investment strategy on IIB for manager 2 under RPC, RRAC, RWC, and Merton optimal strategy versus time $t$.

to obtain better relative wealth. We can also find that the magnitude of the increase for manager 2 is lower than that of manager 1. It may be because manager 2 is more risk averse than manager 1 .

Figure 7 studies the situation of the optimal strategy of manager 1 on stocks with respect to the RRAC coefficient $k_{1}$. 


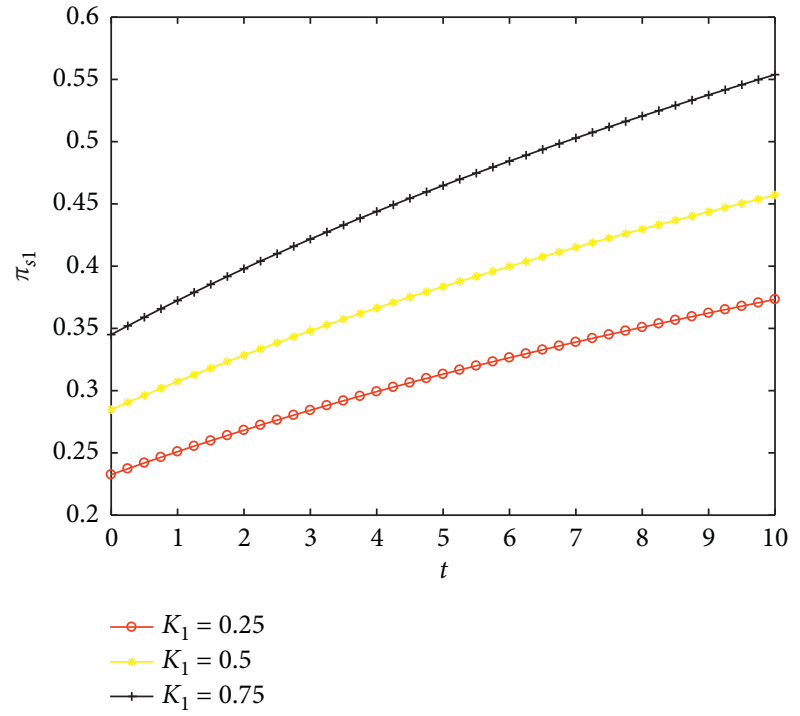

FIgURE 5: Optimal investment strategy on stocks for manager 1 under RWC versus time $t$.

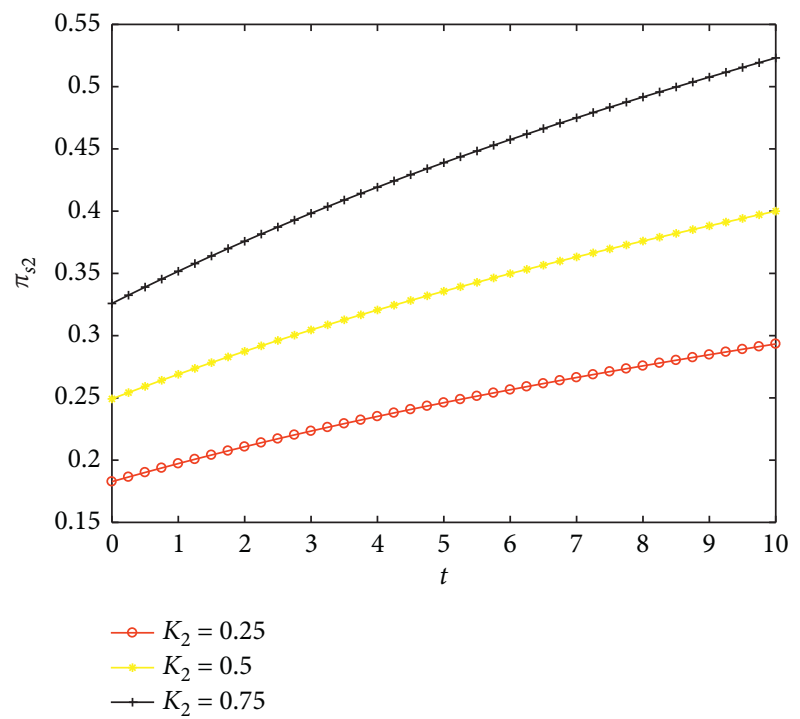

FIGURE 6: Optimal investment strategy on stocks for manager 2 under RWC versus time $t$.

Unlike the case of RWC, in the case of RRAC, the optimal investment ratio of manager 1 on the stock decreases as increasing $k_{1}$. The main reason for this phenomenon is that our manager 1 , as a RRAC investor, is affected by the risk aversion of manager 2 and raises his risk aversion level, which leads to a reduction in the proportion of investment on stock assets. However, the proportion of his investment on stock is constantly raising under different RRAC coefficient. For the same reason, Figure 8 shows that manager 2 gradually increases the proportion of investment on stock assets as the RRAC coefficient increases.

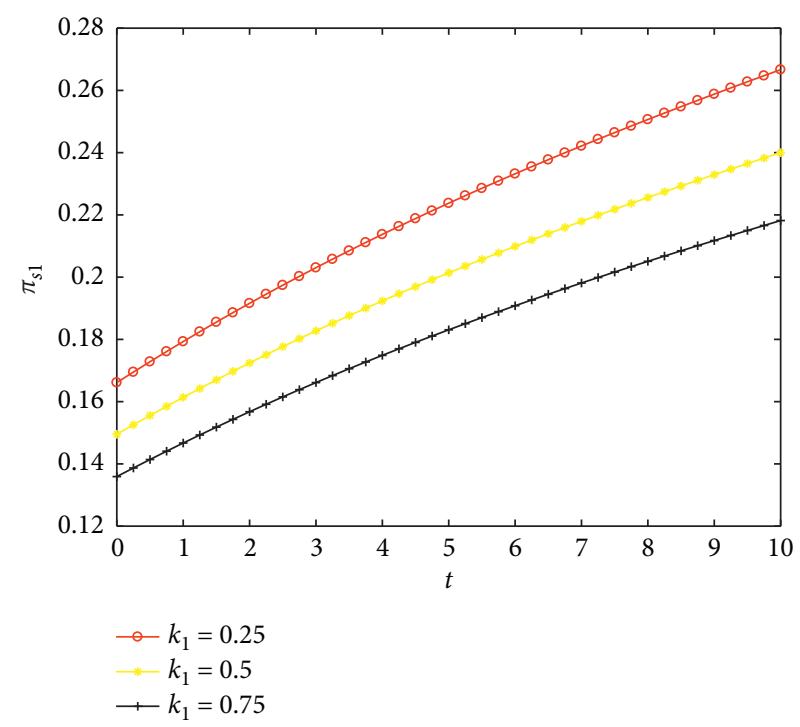

FIGURE 7: Optimal investment strategy on stocks for manager 1 under RRAC versus time $t$.

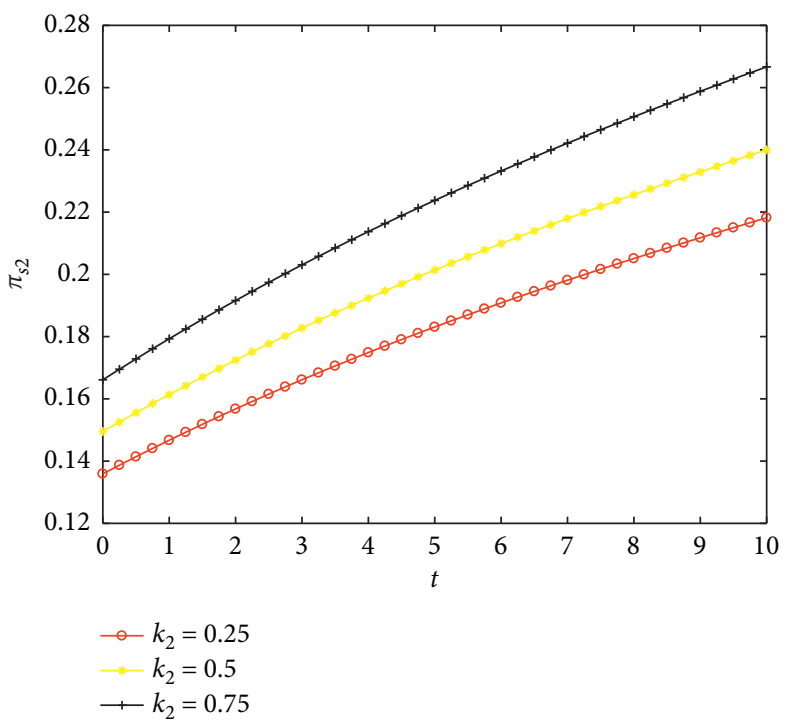

Figure 8: Optimal investment strategy on stocks for manager 2 under RRAC versus time $t$.

\section{Conclusion}

This article discusses the optimal investment strategy adopted by a relative performance concern manager to maximize the expected terminal utility under the risk of inflation background. At the same time, the manager utility function is assumed to be CARA utility, and the stock process is following the Heston model. The opponent that the RRAC manager compares with can be an ordinary pension manager, or a "representative manager" in the 
pension market. Unlike the previous literature, which only focused on the manager's relative wealth concern, we further considered relative risk aversion concern and reached a series of conclusions. Finally, numerical simulation results are given based on the obtained conclusions. Later, we can further focus on the situation where two managers can invest in different stock assets (there is a correlation, or further, a fuzzy correlation between the two stocks). We can even consider the optimal investment portfolio with other background risks, such as interest rate risk and wage risk. In this case, we can draw more general conclusions.

\section{Data Availability}

The data used to support the findings of this study are included within the article.

\section{Conflicts of Interest}

The authors declare that they have no conflicts of interest.

\section{Acknowledgments}

This work was supported by the Scientific Research Foundation of the Higher Education Institutions of Gansu Province under Grant 2017A-139 and Fundamental Research Funds for the Central Universities under Grant JBK2007190.

\section{References}

[1] J. Gao, "Optimal portfolios for DC pension plans under a CEV model," Insurance: Mathematics and Economics, vol. 44, no. 3 , pp. 479-490, 2009.

[2] G. Guan and Z. Liang, "A stochastic nash equilibrium portfolio game between two DC pension funds," Insurance: Mathematics and Economics, vol. 70, pp. 237-244, 2016.

[3] E. Vigna, "On efficiency of mean-variance based portfolio selection in defined contribution pension schemes," Quantitative Finance, vol. 14, no. 2, pp. 237-258, 2014.

[4] J.-F. Boulier, S. Huang, and G. Taillard, "Optimal management under stochastic interest rates: the case of a protected defined contribution pension fund," Insurance: Mathematics and Economics, vol. 28, no. 2, pp. 173-189, 2001.

[5] Y. Zeng, D. Li, Z. Chen, and Z. Yang, "Ambiguity aversion and optimal derivative-based pension investment with stochastic income and volatility," Journal of Economic Dynamics and Control, vol. 88, pp. 70-103, 2018.

[6] N.-w. Han and M.-w. Hung, "Optimal asset allocation for DC pension plans under inflation," Insurance: Mathematics and Economics, vol. 51, no. 1, pp. 172-181, 2012.

[7] G. Guan and Z. Liang, "Optimal management of DC pension plan in a stochastic interest rate and stochastic volatility framework," Insurance: Mathematics and Economics, vol. 57, pp. 58-66, 2014.

[8] T. Veblen, The Theory of the Leisure Class: An Economic Study of Institutions, The Modern Library, New York, NY, USA, 1899.
[9] A. B. Abel, Asset Prices under Habit Formation and Catching up with the Joneses, National Bureau of Economic Research, Cambridge, MA, USA, 1990.

[10] J. Gali, "Keeping up with the Joneses: consumption externalities, portfolio choice, and asset prices," Journal of Money, Credit and Banking, vol. 26, no. 1, pp. 1-8, 1994.

[11] J.-P. Gómez, "The impact of keeping up with the Joneses behavior on asset prices and portfolio choice," Finance Research Letters, vol. 4, no. 2, pp. 95-103, 2007.

[12] S. Browne, "Stochastic differential portfolio games," Journal of Applied Probability, vol. 37, no. 1, pp. 126-147, 2000.

[13] G.-E. Espinosa and N. Touzi, "Optimal investment under relative performance concerns," Mathematical Finance, vol. 25, no. 2, pp. 221-257, 2015.

[14] S. Basak and D. Makarov, "Competition among portfolio managers and asset specialization," SSRN Journal, 2015.

[15] G. Guan and Z. Liang, "Optimal management of DC pension plan under loss aversion and value-at-risk constraints," Insurance: Mathematics and Economics, vol. 69, pp. 224-237, 2016.

[16] M. J. Brennan and Y. Xia, "Dynamic asset allocation under inflation,” The Journal of Finance, vol. 57, no. 3, pp. 1201-1238, 2002.

[17] M. Kwak and B. H. Lim, "Optimal portfolio selection with life insurance under inflation risk," Journal of Banking \& Finance, vol. 46, pp. 59-71, 2014.

[18] J. Sun, Z. Li, and Y. Li, "Equilibrium investment strategy for DC pension plan with inflation and stochastic income under Hestons SV model," Mathematical Problems in Engineering, vol. 2016, Article ID 2391849, 18 pages, 2016.

[19] B. S. Lee, "Stock returns and inflation revisited: an evaluation of the inflation illusion hypothesis," Journal of Banking \& Finance, vol. 34, no. 6, pp. 1257-1273, 2010. 